\title{
A novel miniature coplanar band-pass filter for ISM applications
}

\author{
Badr Nasiri ${ }^{1}$, Abdelhadi Ennajih ${ }^{2}$, Ahmed Errkik $^{3}$, Jamal Zbitou $^{4}$, Mounir Derri ${ }^{5}$ \\ 1,2,3,4 LMEET, FST of Settat, Hassan 1st University, Settat, Morocco \\ ${ }^{5}$ LAGES, Hassania School of Public Works, Casablanca, Morocco
}

\section{Article Info \\ Article history: \\ Received Oct 3, 2018 \\ Revised Jan 7, 2019 \\ Accepted Jul 22, 2019}

\section{Keywords:}

Coplanar

Filter

ISM

Metamaterial

Split ring resonator

\begin{abstract}
This paper presents a novel approach to design a compact miniature coplanar band-pass filter by using rectangular split ring resonator. This proposed circuit is designed for the Industrial, Scientific and, Medical (ISM) frequency band applications at $2.4 \mathrm{GHz}$. At the first stage, a metamaterial resonator is designed and simulated in a TEM waveguide to verifiy its electromagnetic proprieties around the desired frequency bands. At the second stage, a band pass filter is designed using the proposed metamaterial resonator. Many parametric studies are realized to investigate the effect and influence of some resonator parameters on the proposed BPF performances. ADS Agilent and CST-MWS solvers are used in order to verify the simulated results. The circuit frequency responses show an excellent insertion loss and good return loss in the passband.
\end{abstract}

This is an open access article under the $\underline{C C B Y-S A}$ license.

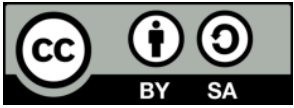

Corresponding Author:

BadrNasiri,

Laboratory of Mechanic, Informatics, Electronic and Telecommunication,

Hassan 1st University,

FST de Settat, Km 3, B.P. : 577 Route de Casablanca, Settat, Morocco.

Email: b.nasiri@uhp.ac.ma

\section{INTRODUCTION}

The modern wireless communication systems and technologies, in particular, GSM, ISM and WiMAX have recently attracted an important attention of many researchers and developers with a view to improve the transmission and/or reception signal quality and, also to propose new compact and miniature devices can be implemented in these systems and used for these technologies[1-4]. Due to its irreplaceable role in the transmission-reception chain, The microwave filters have widely studied in order to achieve a miniature circuit size [5-8], excellent attenuation in rejected bands and low insertion loss in the desired range of frequencies [9-12], which led to create novel approaches and new resonators can be implemented in filter structures for achieving these characteristics simultaneously. Because these requirements are hard to achieve by the classical and conventional techniques [13-16].

Newly, the unusual electromagnetic proprieties of metamaterial resonators are opening new possibilities to obtain small microwave components such as antennas, power dividers and, filters. Moreover, good studies and uses of metamaterials give us new circuits with excellent electrical performances [17-19]. Generally, the natural materials have positive effective medium parameters. On the other hand, the split ring resonator metamaterial have the capability to exhibit negative effective permeability around their resonance frequencies as it is presented by Pendry in 1999 [20], and the complementary SRRs characterized by negative effective permittivity. Besides, the artificial left-handed metamaterials have negativepermeabilitty, permitivitty and, refractive indice, which do not exist in naturalmaterials [21-25]. This letter describes a novel coplanarbandpass filter based on SRR metamaterial 
which is operating in the ISM band that is corresponding to the $2.4 \mathrm{GHz}$ frequency. A brief study is presented in order to investigate the resonator resonant frequency and its electromagnetic proprieties. The proposed circuit is characterized by a good bandwidth matching and an excellent rejection in both attenuated bands.

\section{METAMATERIAL STRUCTURE AND ITS CHARACTERISTICS}

Among the resonators that have a positive effect on the miniaturization of the microwave circuit and which have a magnetic response, we find the rectangular-SRR. This type of resonator is generally formed by two concentric enclosed rectangular rings with two slits in opposite sides. Its resonant frequency is given by (1). This frequency can be controlled by the resonator parameters. Whereas the global capacity $\mathrm{C}_{\mathrm{R}-\mathrm{SRR}}$ is due to the two splits and space between rectangular rings, and the global inductance $\mathrm{L}_{\mathrm{R}-\mathrm{SRR}}$ is resulting from width and length of the rings. The geometrical configuration parameters of the resonator which will be used in our proposed design are presented in Figure 1. Its dimensions are chosen to obtain a resonant frequency in the desired frequency range.

$$
f=\frac{1}{2 \pi \sqrt{L_{R-S R R} C_{R-S R R}}}
$$

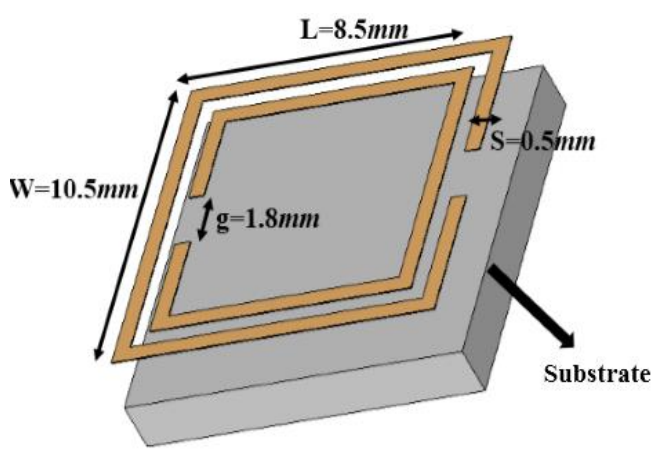

(a)

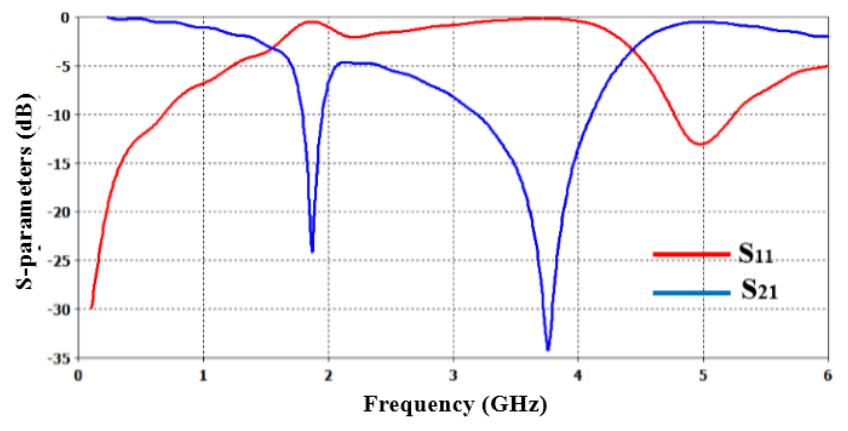

(b)

Figure 1. (a) Metamaterial resonator, (b) Its simulation results

The existence of the special electromagnetic proprieties canbe verified and carried outusing (4) and (5). Figure 2 illustrates the extracted real and imaginary parts of the permeability of the metamaterial resonator.

$$
n=\frac{1}{k_{0}} \cos ^{-1}\left[\frac{1}{2 S_{21}}\left(1-S_{11}^{2}+S_{21}^{2}\right)\right]
$$




$$
\begin{aligned}
& z= \pm \sqrt{\frac{\left(1+S_{11}\right)^{2}+S_{21}^{2}}{\left(1-S_{11}\right)^{2}+S_{21}^{2}}} \\
& \varepsilon_{\text {eff }}=\frac{n}{z} \\
& \mu_{\text {eff }}=n z
\end{aligned}
$$

Where; $n=$ medium refractive index

$z=$ medium impedance

$k_{0}=$ wa ve number equivalent to $2 \pi / \lambda 0$

$d=$ thickness of the substrate of R-SRR

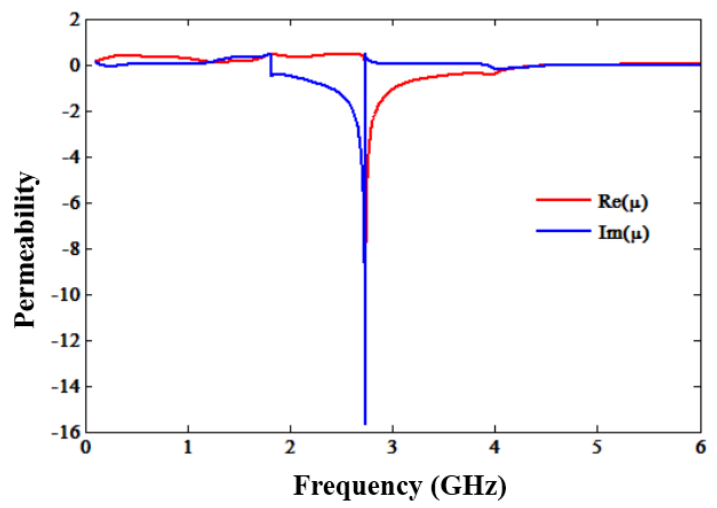

Figure 2. Metamaterial effective permeability versus frequency

\section{ANALYSIS AND DESIGN PROCEDURE OF THE BAND-PASS FILTER}

With a view to meet the requirement of the structure size and transmission or/and reception features, various optimization technics are used by two electromagnetic solvers and several simulated results are carried out and analyzed so as to determine the best one which satisfies the band-pass filter specifications. For the purpose of achieving a pass-band response, the chosen metamaterial resonator is implemented and connected with 50 coplanar feeding lines in order to facilitate its integration with other planar components. The optimized filter is shown in the Figure 3. In orther to investigate the effect of the metamaterial unit cell on the circuit behavior, we changed the length value of the used cells from $6.5 \mathrm{~mm}$ to $9.5 \mathrm{~mm}$ and keept all other parameters invariant. As we can see in Figures 3 and 4, as we increase the length of the resonator, the centre frequency of the bandwidth decreases, which allows us to say that the transmission band of this proposed filter can be shifted and controlled by L-resonator.

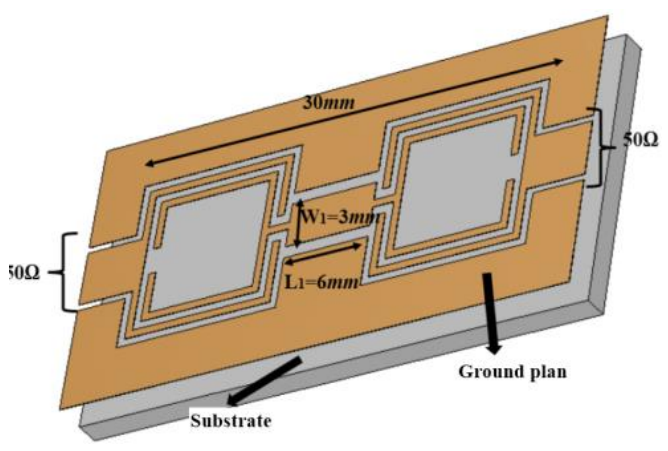

Figure 3. Proposed coplanarband-pass filter 

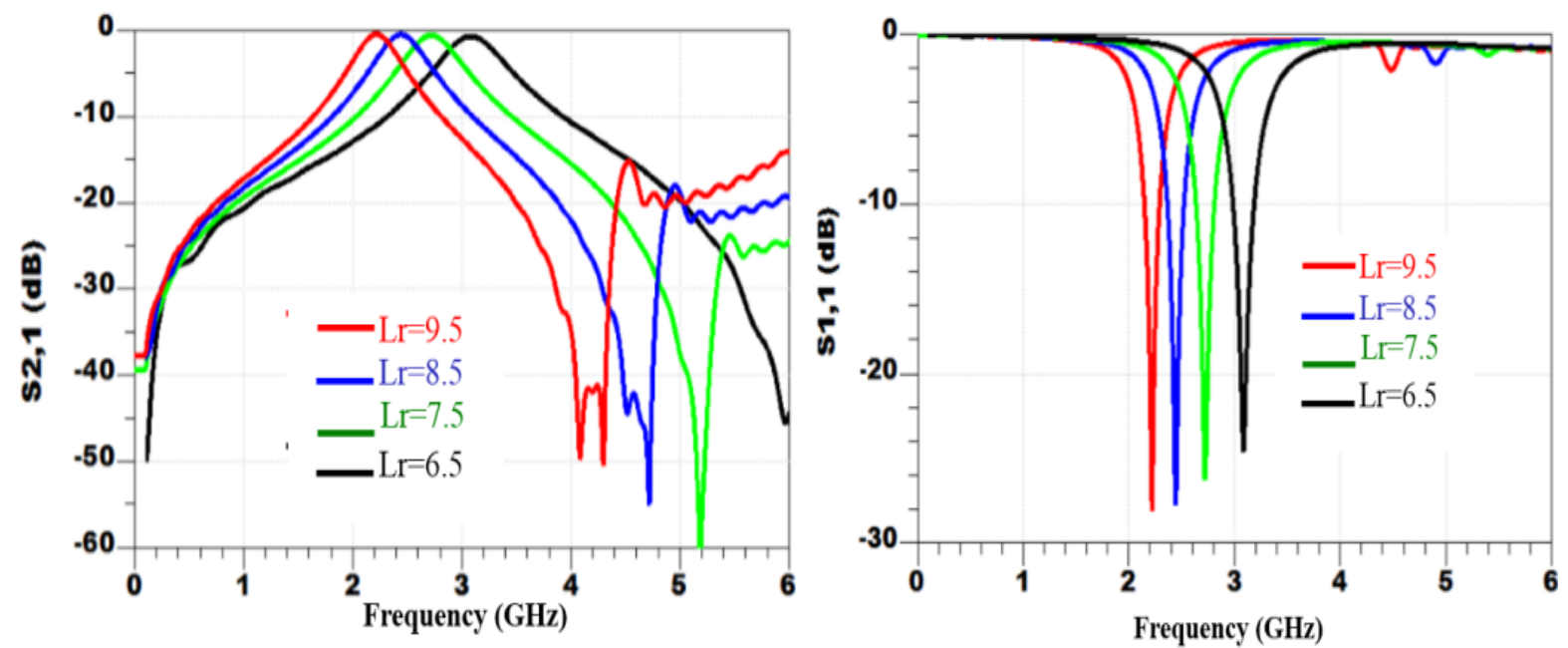

Figure 4. Effect of cell length on the designed bandpass filter

The centre frequency of the bandwidth of the designed filter can be shifted from $3.4 \mathrm{GHz}$ to $2 \mathrm{GHz}$ by varying the width of the resonator, which allows us to conclude that the desired frequency band of our bandpass filter can be controlled by changing the parameters of the metamaterial resonator as shown in the Figure 5.
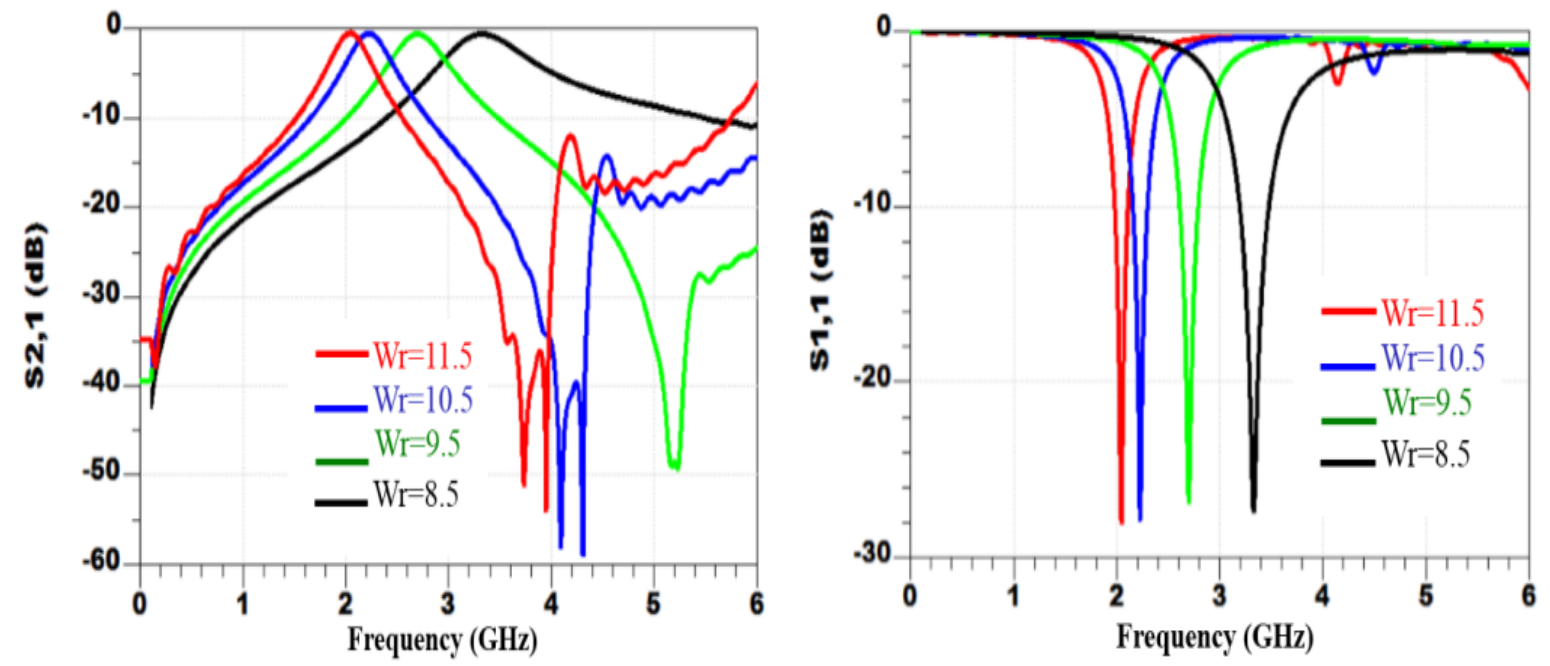

Figure 5. Effect of cell width on the designed bandpass filter

The results of the final circuit in terms of reflection and transmission coefficients are shown in Figure 6. The obtained results show a bandpass behavior with a centre frequency of $2.4 \mathrm{GHz}$ (ISM band). An excellent adaptation and a good level of attenuation are achieved in both rejection bands. In order to verify the CST Microwave simulation results, another simulation is performed via Agilent ADS. From the Figure below, we deduce that there is a good agreement between the two simulations. The slight deviations which we can observe in bandwidth can be explained by the different methods and different numerical resolutions used in Agilent ADS and CST MWS software.

The surface current distribution of the proposed circuit at the central frequency $2.4 \mathrm{GHz}$ of the bandwidth and at $4.5 \mathrm{GHz}$ of the second rejected band is illustrated in Figure 7 . We can notice that we achieve a maximum transmission of radiofrequency power in the ISM band and a good rejection level in the attenuated bands. 


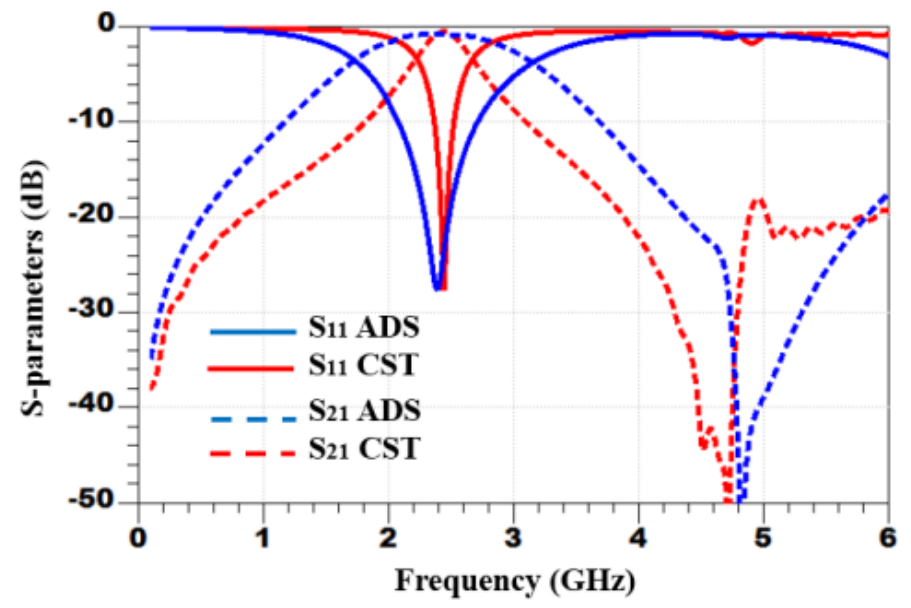

Figure 6. Simulation results by CST-MWS and ADS agilent
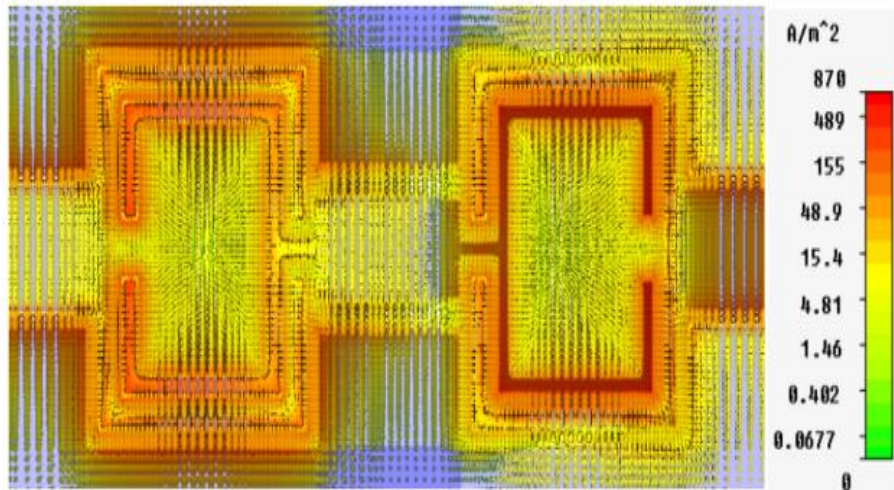

(a)

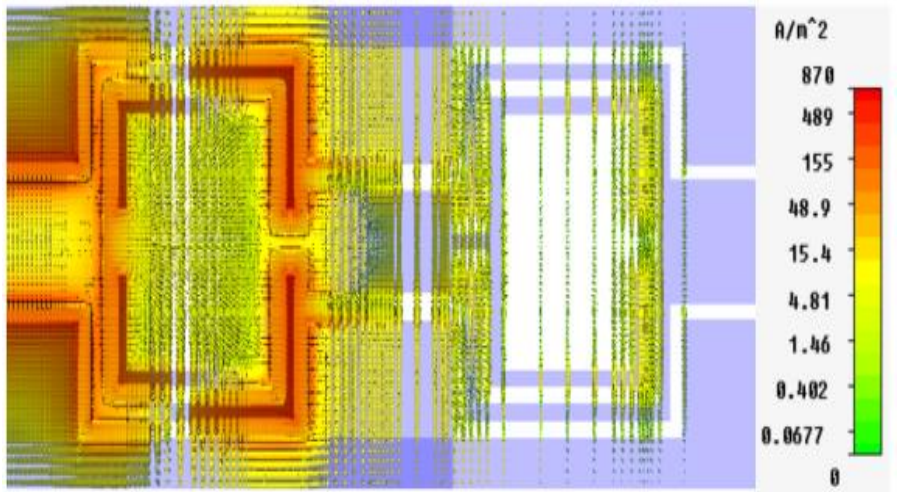

(b)

Figure 7. Surface current density (a) At the frequency $2.4 \mathrm{GHz}$, (b) At the frequency $4.5 \mathrm{GHz}$

This proposed bandpass filter circuit is compared with other previously published works in the literature. Table 1 shows that our structure has excellent passband characteristics and good attenuated band performances. Most importantly, it has a miniature and compact size compared to its operating frequency range and it is characterized by a fractionalbandwidth of $\mathrm{FWB}=16 \%$. 


$$
F B W=\frac{f_{2}-f_{1}}{f_{0}} 100 \%
$$

Table 1. Comparison among published works and the proposed filter

\begin{tabular}{ccccc}
\hline $\begin{array}{c}\text { Parameters/ } \\
\text { Reference }\end{array}$ & $\begin{array}{c}\text { Pass-band } \\
(\mathrm{GHz})\end{array}$ & FWB & $\begin{array}{c}\text { S11 } \\
(\mathrm{dB})\end{array}$ & $\begin{array}{c}\text { Size } \\
\left(\mathrm{mm}^{2}\right)\end{array}$ \\
\hline$[9]$ & {$[2,2.2]$} & $9.5 \%$ & $20 \mathrm{~dB}$ & 795.5 \\
{$[12]$} & {$[4.6,4.8]$} & $4.25 \%$ & $25 \mathrm{~dB} \geq$ & $1200 \geq$ \\
The proposed Filter & {$[2.2,2.6]$} & $16 \%$ & $25 \mathrm{~dB} \geq$ & 480 \\
\hline
\end{tabular}

\section{CONCLUSION}

A novel coplanar band-pass filter is designed on an FR-4 substrate and it is achieved by using two metamaterial cells. The proposed circuit is validated by two electromagnetic solvers and good agreement is obtained between both simulated results. the filter is operating in the ISM band, low insertion loss which is less than $0.2 \mathrm{~dB}$ is obtained in the bandwidth and good power rejection level is attained in two undesired bands. This filter has small and miniature size and high electrical performances whichallow us to say that this circuit is a good choice in modern communication systems.

\section{ACKNOWLEDGEMENTS}

We thank Mr. Mohamed Latrach Professor in ESEO, engineering institute in Angers, France, for allowing us to use all the equipment and solvers a vailable in his laboratory

\section{REFERENCES}

[1] N. A. Wahab, I. Pasya, M. F. A. Khalid, I. M. Yassin, S. H. Herman, Z. Awang, "Pseudo-elliptic bandpass filters using closed-loop resonator," International Journal on Advanced Science, Engineering and Information Technology, vol. 7, no. 2, pp. 345-351, March 2017.

[2] A. Ennajih, J. Zbitou, M. Latrach, A. Errkik, and R.Mandry, "A new dual band printed metamaterial antenna for RFID reader applications," International Journal of Electrical and Computer Engineering, vol. 7, no.6, pp. 3507-3514, December 2017.

[3] A. Auob, and L. Ali, "Compact lowpass filter with wde stop-band using open stubs loaded spiral microstrip resonant cell," Aces Journal, vol. 28, no.1, pp. 27-34, Jan. 2013.

[4] K. Jang, S. kahng, J. Jeon, Q. Wu, "Compact dual-band three-way metamaterial power divider with a hybrid CRLH phase-shift line," Journal Of Electromagnetic Engineering and Science, vol. 14, no.1, pp.15-24, 2014.

[5] A. Ennajih, J. Zbitou, M. Latrach, A. Errkik, and R.Mandry, "New miniature microstrip antenna based on metamaterial for RFID applications," TELKOMNIKA (Telecommunication Computing Electronics and Control), vol. 16, no.1, pp. 174-181, Feb 2018

[6] A. Boutejdar, "A new approach to design compact tunable bpf starting from simple lpf topology using a single t-dgs-resonator and ceramic capacitors," Microwave and Optical Technology Letters, vol. 58, no.5, pp. 1142-1148, 2016.

[7] S. Mao and Y. Chueh, "Coplanar waveguide bandpass filters with compact size and wide spurious-free stopband using electromagnetic bandgap resonators," in IEEE Microwave and Wireless Components Letters, vol. 17, no. 3, pp. 181-183, March 2007.

[8] E. S. Ahmed, "Compact dual-band coupled T-shaped SIR filter for WLAN applications," TELKOMNIKA (Telecommunication Computing Electronics and Control), vol. 15, no. 4, pp. 1677-1681, Dec. 2017.

[9] M. Mabrouk, Z. Zakaria, Y. E. Masrukin, T. Sutikno, A. R. Othman, N. Edward, "Switchable dual-band bandpass filter based on stepped impedance resonator with u-shaped defected microstrip structure for wireless applications," TELKOMNIKA (Telecommunication Computing Electronics and Control), vol. 17, no. 2, pp. 1032-1039, April. 2019.

[10] Y. Gaurav, R.K. Chauhan " A compact UWB BPF with a notch band using rectangular resonator sandwiched between interdigital structure," International Journal of Electrical and Computer Engineering, vol. 7, no. 5, pp. 2420-2425, Oct. 2017.

[11] F. Wei, P. Qin, Y. J. Guo and X. Shi, "Design of multi-band bandpass filters based on stub loaded stepped-impedance resonator with defected microstrip structure," in IET Microwaves, Antennas \& Propagation, vol. 10, no. 2, pp. 230-236, 2912016.

[12] B. Nasiri, A. Errkik, J. Zbitou, A. Tajmouati, L. El Abdellaoui, M. Latrach, "A new compact and wide-band band-stop filter using rectangular SRR," TELKOMNIKA (Telecommunication Computing Electronics and Control), vol. 16, no.1, pp. 110-117, Feb. 2018. 
[13] F. Martin, F. Falcone, J. Bonache, R. Marques and M. Sorolla, "Miniaturized coplanar waveguide stop band filters based on multiple tuned split ring resonators," in IEEE Microwave and Wireless Components Letters, vol. 13, no. 12, pp. 511-513, Dec. 2003.

[14] J. Pu, F. Xu, and S. Qiu, "A miniaturized coplanar waveguide band pass filter using novel single split-ring resonators," in IEEE MTT-S International Wireless Symposium, 2016. IWS 2016, pp. 1-3, 2016.

[15] C. Máximo-Gutiérrez, J. Hinojosa, A. Alvarez-Melcon, "Design of wide band-pass substrate integrated waveguide (SIW) filters based on stepped impedances," AEU-International Journal of Electronics and Communications, vol. 100, pp. 1-8, February 2019.

[16] D. Lu, Y. Yu, M.-C. Tang, T. Shi, "Design of compact wideband bandpass filter with broad stopband using hybrid HMSIW and open-circuit tri-section stepped impedance resonators," Microwave and Optical Technology Letters, vol. 60, no.12, pp. 2998-3003, Decembre 2018.

[17] B. Yang, H. Qian, and X. Luo, "Compact CPW bandpass filter with ultra-wide stopband using slow-wave structure," in IEEE International Workshop on Electromagnetics: Applications and Student Innovation Competition, 2016. (iWEM), pp. 1-3, 2016.

[18] J. Carver, V. Reignault, F. Gadot, " Engineering of the metamaterial-based cut-band filter," Applied Physics A, vol.117, no.2, pp.513-516, 2014.

[19] B. Nasiri, A. Errkik, J. Zbitou, A. Tajmouati, L. El Abdellaoui, M. Latrach, "A miniature microstrip bsf using complementary split ring resonator," International Journal of Intelligent Engineering and Systems, vol. 11, no. 3, pp. 29-36, June 2018.

[20] J. B. Pendry, A. J. Holden, D. J. Robbins and W. J. Stewart, "Magnetism from conductors and enhanced nonlinear phenomena," in IEEE Transactions on Microwave Theory and Techniques, vol. 47, no. 11, pp. 2075-2084, Nov. 1999.

[21] B. Nasiri, A. Errkik, J. Zbitou, A. Tajmouati, L. El Abdellaoui, M. Latrach, " A compact miniature microstrip low pass filter based on dumbbell dgs and srr unit cell," International Journal of Microwave and Optical Technology, vol. 13, no.1, pp. 32-39, January 2018.

[22] B. Nasiri, A. Errkik, J. Zbitou, A. Tajmouati, L. El Abdellaoui, M. Latrach, " A compact planar low-pass filter based on metamaterial," International Journal of Electrical and Computer Engineering, vol. 8, no.6, pp. 4972-4980, December 2018.

[23] J. B. Pendry, A. J. Holden, and W. J. Stewart, "Extremely low frequency plasmons in metallic mesostructures," Phys. Rev. Lett., vol. 76, pp. 4773-4776, 1996.

[24] V. G. Veselago," The electrodynamics of substances with simultaneous negative values of $\varepsilon$ and $\mu$," IEEE Soviet Physics Uspekhi, vol. 10, pp. 509-514, 1968.

[25] R. Marques, F. Mesa, J. Martel and F. Medina, "Comparative analysis of edge- and broadside- coupled split ring resonators for metamaterial design - theory and experiments," in IEEE Transactions on Antennas and Propagation, vol. 51, no. 10, pp. 2572-2581, Oct. 2003. 